\title{
PARÂMETROS ESPECTRAIS DA VOZ EM CRIANÇAS RESPIRADORAS ORAIS
}

\section{Spectral parameters on voices of mouth-breathing children}

\author{
Danieli Viegas ${ }^{(1)}$, Flávia Viegas ${ }^{(2)}$, Ciríaco Cristovão Tavares Atherino ${ }^{(3)}$, Heidi Elisabeth Baeck ${ }^{(4)}$
}

\section{RESUMO}

Objetivo: investigar parâmetros espectrais ( $f_{0}$ e formantes) descritivos da voz de crianças respiradoras orais, visando contribuir na caracterização desta população e, consequentemente, na efetividade do diagnóstico. Métodos: foram selecionadas 50 crianças respiradoras orais e 101 crianças com respiração nasal de ambos os gêneros entre cinco e dez anos. Os sinais de fala foram obtidos a partir das gravações de sentenças-veículo e os segmentos das vogais orais [a], [i] e [u] do português falado no Brasil em posição tônica foram selecionados para a estimação da frequência fundamental $\left(f_{0}\right)$ e da frequência dos três primeiros formantes $\left(F_{1}, F_{2} \in F_{3}\right)$. O software Praat foi utilizado para gravar, recortar e processar os sinais. Resultados: a frequência fundamental das vogais [i] e [u] apresentouse menor nos respiradores orais, enquanto que a frequência de $F_{1}$ da vogal [u] foi mais elevada no mesmo grupo. Conclusão: a partir dos resultados expostos, concluiu-se que a frequência fundamental consiste em um parâmetro vocal que auxilia na diferenciação de crianças respiradores orais e respiradoras nasais, contribuindo, desta forma para uma caracterização mais efetiva do respirador oral.

DESCRITORES: Respiração Oral; Voz; Processamento de Sinais Assistido por Computador; Criança; Acústica da Fala

\section{INTRODUÇÃO}

A Síndrome da Respiração Oral (SRO) vem sendo descrita como um dos mais preocupantes problemas de saúde pública ${ }^{1,2}$ que acomete a população infantil ${ }^{3}$. No momento em que o padrão respiratório nasal é inadequadamente substituído por um padrão oral ou oronasal podem ocorrer

(1) Fonoaudióloga da Prefeitura da Cidade do Rio de Janeiro, PCRJ, Rio de Janeiro, RJ; Mestre em Fonoaudiologia pela Universidade Veiga de Almeida.

(2) Fonoaudióloga da Prefeitura da Cidade do Rio de Janeiro, PCRJ, Rio de Janeiro, RJ; Mestre em Fonoaudiologia pela Universidade Veiga de Almeida.

(3) Médico; Professor Adjunto do Mestrado Profissionalizante em Fonoaudiologia da Universidade Veiga de Almeida, UVA, Rio de Janeiro, RJ; Professor Adjunto da disciplina de Otorrinolaringologia da Faculdade de Ciências Médicas da Universidade Estadual do Rio de Janeiro, UERJ, Rio de Janeiro, RJ; Doutor em Otorrinolaringologia pela Faculdade de Medicina da Universidade de São Paulo.

(4) Fonoaudióloga; Professora Adjunta do Mestrado Profissionalizante em Fonoaudiologia da Universidade Veiga de Almeida, UVA, Rio de Janeiro, RJ; Doutora em Engenharia Biomédica pela Universidade Federal do Rio de Janeiro.

Conflito de interesses: inexistente prejuízos na postura corporal e, em especial, na morfologia craniofacial ${ }^{4-7}$. Autores referem que o grau de comprometimento dos diversos componentes estruturais irá depender, além da predisposição genética, da idade e do tempo em que a respiração oral persiste ${ }^{2,8-11}$. A primeira década de vida foi apontada como sendo o período mais susceptível, no qual podem ocorrer mudanças consideráveis na morfologia craniofacial ${ }^{9,12}$. Assim sendo, o restabelecimento mais breve possível da respiração nasal é imprescindível para prevenir ou minimizar os efeitos deletérios causados pela respiração oral ${ }^{10}$.

Diversas formas têm sido buscadas para descrever o respirador oral em toda sua complexidade, gerando um conhecimento multifacetado no que se refere aos aspectos que caracterizam este modo de respiração. Alguns pesquisadores basearam o diagnóstico da respiração oral na avaliação otorrinolaringológica ${ }^{6,12-14}$, outros em testes específicos como o 'espelho de Glatzel' e o 'preenchimento da cavidade oral com água' 1,2,11, porém grande parte dos estudos caracterizou o respirador oral a partir de seus sinais e sintomas ${ }^{9,10,15,16}$. É possível observar que as pesquisas que abordam os sinais 
e sintomas do respirador oral caminham na mesma direção, investigando prioritariamente alterações estruturais e funcionais do sistema estomatognático. Mesmo entre os estudos mais recentes ${ }^{7,10,14-17}$ observam-se descrições de sinais e sintomas relacionados prioritariamente à motricidade orofacial do respirador oral. Disfonias vêm sendo mencionadas por alguns autores como parte do quadro, porém não têm sido amplamente investigadas.

Recentemente, um estudo que pretendeu realizar uma extensa revisão teórica sobre respiração oral e disfonia concluiu que o tema é escasso, com poucos artigos que exploram a correlação sistemática entre as duas patologias ${ }^{18}$. Dentre os poucos estudos encontrados na literatura, grande parte é relativamente atual, publicado após o ano $2000{ }^{19-24}$. Em alguns trabalhos a investigação da qualidade vocal foi baseada em dados de anamnese e avaliações perceptivo-auditivas ${ }^{19,21,22,24}$ enquanto que em outros, a mesma foi baseada em análises espectrográficas ${ }^{20,23}$. De forma geral, o objeto de pesquisa dos autores citados anteriormente não foi a "respiração oral/nasal" propriamente dita, e sim situações análogas promovidas por alergias respiratórias ${ }^{19,22,24}$, obstrução nasal ${ }^{23}$ e patologias como hipertrofia de tonsilas palatinas ${ }^{20}$. A presença de rouquidão, hiponasalidade, alterações da voz ao longo do dia e voz fraca foram mencionadas como características nos quadros de rinite alérgica em adultos ${ }^{19}$ e em crianças ${ }^{22}$. A alergia respiratória também foi apontada como um fator de risco para sintomas vocais, pois pode causar muco excessivo e edema nas pregas vocais ${ }^{24}$. Dois estudos internacionais investigaram a qualidade vocal de adultos utilizando a análise acústica, antes e após a realização de um procedimento (tonsilectomia e uso de descongestionante nasal), situações que corresponderiam à respiração oral e nasal, respectivamente ${ }^{20,23}$. Os resultados apontaram que não houve diferença significante na qualidade vocal nas duas situações. O único trabalho encontrado na literatura, que investigou especificamente respiradores orais infantis, utilizou análise perceptivo-auditiva e apontou diferenças na qualidade vocal de acordo com a etiologia da respiração oral ${ }^{21}$.

A carência de dados na literatura evidencia a necessidade de estudos que investiguem de forma abrangente e aprofundada os parâmetros vocais em respiradores orais. Desta forma, o presente estudo teve por objetivo investigar medidas acústicas vocais (frequência fundamental e frequência dos três primeiros formantes) em crianças respiradoras orais, na faixa de cinco a dez anos, visando contribuir para a caracterização desta população e, consequentemente, na efetividade do diagnóstico.

\section{MÉTODOS}

A amostra foi constituída por 453 segmentos vocais (três segmentos por criança) de 151 crianças entre cinco e dez anos, sendo 50 crianças respiradoras orais e 101 crianças com respiração nasal (grupo controle), selecionadas por uma sequência de procedimentos (pré-seleção e seleção propriamente dita).

O número de sujeitos no grupo de respiradores orais foi distribuído por faixa etária e gênero da seguinte forma: 10 crianças entre 5 anos e 5 anos e 11 meses, sendo 2 meninas e 8 meninos; 9 crianças entre 6 anos e 6 anos e 11 meses, 2 meninas e 7 meninos; 15 crianças entre 7 anos e 7 anos e 11 meses, 4 meninas e 11 meninos; 8 crianças entre 8 anos e 8 anos e 11 meses, sendo 2 meninas e 6 meninos; 6 crianças entre 9 anos e 9 anos e 11 meses, todas do gênero masculino e 2 crianças entre 10 anos e 10 anos e 11 meses, ambas do gênero feminino. $O$ grupo controle foi pareado ao grupo de respiradores orais em gênero e idade. Detalhes dos procedimentos de composição da amostra encontram-se relatados a seguir.

Crianças com suspeita de respiração oral foram pré-selecionadas nos setores de pediatria e odontologia de um Posto de Saúde da cidade do Rio de Janeiro seguindo os critérios: a) ter idade entre cinco e dez anos e b) apresentar frequentemente duas ou mais das seguintes queixas respiratórias: congestão nasal, respirar pela boca durante o dia e/ou noite, roncos, sialorréia noturna, respiração ruidosa durante o repouso e, cansaço frequente ao realizar atividades físicas.

A pré-seleção de crianças respiradoras nasais foi realizada por professores de duas escolas, seguindo os critérios: a) ter idade entre cinco e dez anos e b) não apresentar as queixas respiratórias descritas anteriormente. Os professores distribuíram um questionário aos pais (Figura 1), orientando-os a devolvê-lo preenchido no prazo de uma semana. Foram distribuídos 170 questionários, porém somente 132 foram devolvidos aos professores. Dos questionários devolvidos, dez apresentaram erros na marcação das respostas, como: duas respostas assinaladas para o mesmo item ou item sem marcação. As 122 crianças restantes passaram para a fase de seleção.

As crianças pré-selecionadas tanto pelos professores quanto pelos médicos e odontólogos foram encaminhadas ao setor de fonoaudiologia do referido posto de saúde, onde foram submetidas aos seguintes procedimentos, que resultaram na inclusão ou não na amostra: 1) entrevista com os pais (Figura 1); 2) teste de água na boca; 3) avaliação orofacial (apenas para as crianças com 


\section{ENTREVISTA DIRECIONADA A QUEIXAS RESPIRATÓRIAS:}

Nome:

DN: idade atual: Data:

1 - Seu filho fica com o nariz entupido frequentemente?
( ) sim
( ) não

2 - Seu filho respira com a boca aberta durante o dia?
( ) $\operatorname{sim}$
( ) não

3 - Quando seu filho respira você nota algum ruído?
( ) $\operatorname{sim}$
( ) não

4 - Seu filho dorme de boca aberta?
( ) $\operatorname{sim}$
( ) não

5 - Seu filho baba durante a noite?
( ) $\operatorname{sim}$
( ) não

6 - Seu filho ronca?
( ) $\operatorname{sim}$
( ) não

7 - Seu filho realiza alguma atividade física?
( ) $\operatorname{sim}$
( ) não
Qual?

8 - Seu filho se cansa ao realizar exercícios físicos?
( ) sim
( ) não

Figura 1 - Entrevista com os pais direcionada a queixas respiratórias

suspeita de respiração oral) (Figura 2). A entrevista direcionada a queixas respiratórias teve o objetivo de confirmar ou descartar as referidas queixas e sua frequência. No teste de água na boca a criança foi orientada a manter um gole de água na cavidade oral por três minutos ${ }^{1,2}$, com o objetivo de verificar a capacidade de manutenção da respiração nasal. O protocolo de avaliação orofacial consiste em um conjunto de observações clínicas das estruturas dos órgãos fonoarticulatórios, sendo que o utilizado na presente pesquisa foi anteriormente aplicado em respiradores orais em estudo científico realizado pelas duas primeiras autoras deste estudo ${ }^{10}$. Tal protocolo consistiu em uma adaptação do protocolo proposto por uma importante pesquisadora na área de motricidade oral ${ }^{15}$. A partir desse protocolo foi criado um escore no qual foi atribuído peso (valor) 1 às respostas que caracterizam o respirador oral e não foi atribuído valor às características correspondentes à normalidade (Figura 3). Das 122 crianças pré-selecionadas pelos professores, foram incluídas no grupo controle apenas as crianças que não apresentaram queixas respiratórias, que conseguiram manter a água na boca por três minutos e que apresentaram faixa etária e gênero equivalentes ao grupo de respiradores orais, totalizando 101 crianças.

$\mathrm{Na}$ amostra de respiradores orais foram incluídas crianças cuja presença e frequência dos sintomas respiratórios foram confirmadas na entrevista, além de não conseguirem manter um gole de água na boca por três minutos e apresentarem um escore $\geq$ 12 na avaliação orofacial. Da amostra inicial de 61 crianças que apresentavam duas ou mais queixas respiratórias frequentemente, 11 crianças foram excluídas por terem passado no teste de água na boca, permanecendo mais do que três minutos com água na cavidade oral e/ou apresentarem escore < que 12 na avaliação orofacial. Durante a fase de seleção, os responsáveis pelas crianças assinaram o Termo de Consentimento Livre e Esclarecido. 


\section{PROTOCOLO DE AVALIAÇÃO OROFACIAL}

Proposto por Andrade e col. $(2005)^{10}$

Nome:

Encaminhado por:

Idade: anos e

Data da avaliação:

Endereço:

Telefone:

\section{EXAME CLÍNICO DOS ÓRGÃOS FONOARTICULATÓRIOS:}

\section{LÁBIOS:}
1.1 - Postura habitual:
( ) abertos
( ) entreabertos
1.2 - Lábio superior (espessura):
( ) normal
( ) fino
( ) grosso
1.3 - Presença de eversão:
( ) $\operatorname{sim}$
( ) não
1.4 - Lábio inferior (espessura):
( ) normal
( ) fino
( ) grosso
1.5 - Presença de eversão:
( ) $\operatorname{sim}$
1.6 - Tônus do lábio superior:
( ) normal
( ) não
1.7 - Tônus do lábio inferior
( ) normal
( ) rígido
( ) flácido
( ) rígido
( ) flácido

\section{BOCHECHAS:}

\section{1 - Altura das bochechas:}

2.2 - Volume das bochechas:

( ) mesma altura

( ) alturas diferentes

2.3 - Tônus da bochecha D:

( ) mesmo volume

( ) volumes diferentes

2.4 - Tônus da bochecha E:

( ) normal

( ) rígido

( ) flácido

( ) normal

( ) rígido

( ) flácido

\section{MÚSCULO MENTUAL:}
3.1 - Tônus:
( ) normal
( ) rígido
( ) flácido

\section{MANDÍBULA:}

4.1 - Postura de repouso mandibular:
( ) normal
( ) aberta
( ) desviada para D
( ) desviada para $\mathrm{E}$

5. LÍNGUA:
5.1 - Tensão:
( ) normal
( ) aumentada
( ) diminuída

\section{PALATO:}
6.1 - Duro:
( ) normal
( ) estreitado
( ) atrésico
( ) largo
( ) baixo
( ) alto

Figura 2 - Avaliação Orofacial

Embora os participantes de ambos os grupos tenham sidos selecionados por conveniência, a definição final da amostra que compôs o grupo controle foi manipulada, de forma que este grupo fosse pareado ao de respiradores orais por gênero e idade. Para cada respirador oral, foram incluídos pelo menos dois participantes com respiração nasal de gênero e faixa etária equivalentes.
Tal manipulação foi realizada com o objetivo de controlar o efeito destas variáveis e obter resultados mais consistentes na estimação dos parâmetros de interesse do presente estudo.

Os sinais de fala foram obtidos a partir de gravações de sentenças-veículo "Fale___ para mim", preenchidas com os vocábulos "pápa", "pípi" e "púpu", de forma a obter segmentos das três vogais 


\begin{tabular}{|c|c|c|c|}
\hline \multicolumn{4}{|c|}{ PONTUAÇÃO ATRIBUÍDA AOS ITENS OROFACIAIS } \\
\hline $\begin{array}{l}\text { Item 1: } \\
1.1- \\
1.2- \\
1.3- \\
1.4- \\
1.5- \\
1.6- \\
1.7- \\
\end{array}$ & $\begin{array}{l}\text { abertos }=1 \\
\text { normal }=0 \\
\operatorname{sim}=1 \\
\text { normal }=0 \\
\text { sim }=1 \\
\text { normal }=0 \\
\text { normal }=0\end{array}$ & $\begin{array}{l}\text { entreabertos }=1 \\
\text { fino }=0 \\
\text { não }=0 \\
\text { fino }=0 \\
\text { não }=0 \\
\text { rígido }=0 \\
\text { rígido }=0\end{array}$ & $\begin{array}{l}\text { grosso }=1 \\
\text { grosso }=1 \\
\text { flácido }=1 \\
\text { flácido }=1\end{array}$ \\
\hline $\begin{array}{l}\text { Item 2: } \\
2.1- \\
2.2- \\
2.3- \\
2.4-\end{array}$ & $\begin{array}{l}\text { mesma altura }=0 \\
\text { mesmo volume }=0 \\
\text { normal }=0 \\
\text { normal }=0\end{array}$ & $\begin{array}{l}\text { rígido }=0 \\
\text { rígido }=0\end{array}$ & $\begin{array}{l}\text { alturas diferentes }=1 \\
\text { volumes diferentes = } 1 \\
\text { flácido }=1 \\
\text { flácido }=1\end{array}$ \\
\hline $\begin{array}{l}\text { Item 3: } \\
3.1-\end{array}$ & normal $=0$ & rígido = 1 & flácido = 0 \\
\hline $\begin{array}{l}\text { Item 4: } \\
4.1-\end{array}$ & normal $=0$ & aberta $=1$ & desviada $\mathrm{D}=0$ \\
\hline $\begin{array}{l}\text { Item 5: } \\
5.1-\end{array}$ & normal $=0$ & aumentada $=0$ & diminuída = 1 \\
\hline $\begin{array}{l}\text { Item 6: } \\
6.1-\end{array}$ & $\begin{array}{l}\text { normal }=0 \\
\text { estreitado }=1\end{array}$ & $\begin{array}{l}\text { atrésico }=1 \\
\text { baixo }=0\end{array}$ & $\begin{array}{l}\text { largo }=0 \\
\text { alto }=1\end{array}$ \\
\hline
\end{tabular}

Figura 3 - Escore dos itens orofaciais

em posição tônica. As emissões foram gravadas em uma sala silenciosa utilizando um notebook marca HP (DV 1000), sistema operacional Windows XP, com auxílio do software Praat (versão 5008) e de um microfone (Shure SM 58) posicionado a 10 $\mathrm{cm}$ dos lábios, num ângulo aproximado de $45^{\circ}$. As gravações foram realizadas em mono canal, com taxa de amostragem de $22.050 \mathrm{~Hz}$ e formato "wave". Os dados adquiridos foram submetidos a procedimentos de pré-processamento e processamento de sinais através do referido software, envolvendo o recorte dos segmentos vocálicos e a estimação da frequência fundamental e a frequência dos três primeiros formantes.

O recorte foi realizado de forma manual, porém houve a preocupação em definir critérios de recorte passíveis de serem implementados em um futuro sistema de recorte automático. Os marcos inicial e final foram definidos nos trechos do sinal onde a energia apresentava-se logo acima e logo abaixo, respectivamente, da marca de $20 \%$ da energia máxima. Tal porcentagem foi estabelecida empiricamente com base em investigação experimental e configura um procedimento conservador no sentido de reduzir a probabilidade de obtenção de trechos não estacionários. As estimações dos parâmetros acústicos investigados foram obtidas utilizando um script (ferramenta do software Praat que permite a programação da sequência de comandos a serem realizados para a obtenção das medidas investigadas). O objetivo do uso de tal ferramenta é a otimização do tempo de estimação dos parâmetros. Deve-se ressaltar, no entanto, que as medidas dos sinais também podem ser obtidas de forma manual, ou seja, clicando em cada botão de acordo com a sequência dos comandos. No script a sequência dos comandos já é programada. O script utilizado nesta pesquisa foi desenvolvido pelos autores da mesma.

Este estudo foi aprovado pelo Comitê de Ética em Pesquisa da Secretaria Municipal de Saúde da Prefeitura da cidade do Rio de Janeiro (número 181/2008).

$\mathrm{Na}$ análise estatística foram utilizados os seguintes testes: qui-quadrado $\left(x^{2}\right)$ e exato de Fisher para verificar a existência de diferenças significantes entre os gêneros e as faixas etárias dos grupos testados (controle e respiradores orais). O teste de Mann-Whitney foi utilizado para comparação das medidas de frequência fundamental e de 
formantes entre os dois grupos. A opção pela utilização de teste não paramétrico foi devido ao fato de que as medidas de frequência não apresentaram distribuição normal (Gaussiana) em decorrência da dispersão dos dados, falta de simetria da distribuição e rejeição da hipótese de normalidade (teste de Shapiro-Wilk). As análises foram realizadas com o auxílio do software Statistical Analysis System v.6.04 (SAS Institute, Inc., Cary, North Carolina). O critério de determinação de significância adotado foi o nível de $5 \%$, ou seja, valor de $p$ menor ou igual a 0,05 .

\section{RESULTADOS}

Os testes exato de Fisher e qui-quadrado apontaram que não existiam diferenças significantes na distribuição de gênero $(p=0,18)$ e faixa etária $(p=0,78)$ entre os dois grupos, portanto as crianças foram reunidas em um grupo de respiradores orais e outro de respiradores nasais independente da idade e do gênero.

Em relação às medidas acústicas investigadas, em função do grande número de estimações obtidas, um total de 1.812 valores numéricos, optou-se por expor diretamente os valores médios referentes às tais medidas. Pelo mesmo motivo, os achados foram divididos em seis tabelas, de acordo com cada vogal, facilitando a visualização direta do comportamento do parâmetro acústico entre os dois grupos.
As Tabelas 1, 3 e 5 mostram os valores de média, desvio-padrão e mediana enquanto as tabelas 2, 4 e 6 mostram os correspondentes níveis descritivos ( $p$ valor) do teste de diferença estatística (teste de Mann-Whitney) entre os grupos experimental e controle.

Não foram observadas diferenças estatisticamente significantes em nenhum parâmetro da vogal [a] entre os dois grupos. Os valores de frequência fundamental da vogal [i] apresentaram-se estatisticamente menores no grupo de respiradores orais quando comparados ao grupo controle. Na vogal [u] foram observadas duas diferenças significantes. A frequência fundamental apresentou-se estatisticamente menor no grupo de respiradores orais, enquanto que a frequência do primeiro formante apresentou valores mais elevados neste grupo em relação ao grupo controle.

\section{DISCUSSÃO}

A presente discussão foi desenvolvida de forma a abordar a seleção da amostra de respiradores orais, os resultados obtidos e uma questão particular associada ao grupo de respiradores orais.

A literatura ainda não apresenta um protocolo validado que permita caracterizar a respiração oral em seus diferentes aspectos. De acordo com o perfil do grupo de pesquisadores (otorrinolaringologistas, odontólogos, fonoaudiólogos) pode ser observado um enfoque em determinadas características a serem investigadas, como obstrução

Tabela 1 - Média, mediana e desvio padrão de frequência fundamental e das frequências dos formantes da vogal [a]

\begin{tabular}{c|ccc|ccc}
\hline \multirow{2}{*}{ Parâmetro } & \multicolumn{3}{|c|}{ Grupo Controle $(\boldsymbol{N}=\mathbf{1 0 1})$} & \multicolumn{3}{c}{ Grupo RO $(\boldsymbol{N}=\mathbf{5 0})$} \\
\cline { 2 - 7 } & Média (Hz) & DP $(\mathbf{H z})$ & Mediana $(\mathbf{H z})$ & Média (Hz) & DP $(\mathbf{H z})$ & Mediana $(\mathbf{H z})$ \\
\hline [a] $f_{0}$ & 245 & 28,0 & 241 & 236 & 26,3 & 233 \\
[a] $F_{1}$ & 979 & 194,1 & 984 & 946 & 126,3 & 957 \\
[a] $F_{2}$ & 1847 & 268,2 & 1816 & 1777 & 223,2 & 1788 \\
[a] $F_{3}$ & 3401 & 314,9 & 3395 & 3310 & 383,8 & 3314 \\
\hline
\end{tabular}

DP: Desvio Padrão

Tabela 2 - Teste de diferença estatística entre as médias dos grupos na vogal [a]

\begin{tabular}{c|c}
\hline Parâmetro & p valor \\
\hline$[a] f_{0}$ & 0,057 \\
[a] $F_{1}$ & 0,20 \\
[a] $F_{2}$ & 0,18 \\
[a] $F_{3}$ & 0,12 \\
\hline
\end{tabular}

Teste de Mann-Whitney 
Tabela 3 - Média, mediana e desvio-padrão da frequência fundamental e das frequências dos formantes da vogal [i]

\begin{tabular}{c|ccc|ccc}
\hline \multirow{2}{*}{ Parâmetro } & \multicolumn{3}{|c|}{ Grupo Controle $(\boldsymbol{N}=\mathbf{1 0 1})$} & \multicolumn{3}{c}{ Grupo RO $(\mathbf{N}=\mathbf{5 0})$} \\
\cline { 2 - 7 } & Média (Hz) & DP (Hz) & Mediana (Hz) & Média (Hz) & DP (Hz) & Mediana (Hz) \\
\hline$[i] f_{0}$ & 272 & 34,3 & 271 & 259 & 24,8 & 257 \\
{$[i] F_{1}$} & 333 & 48,9 & 324 & 360 & 214,2 & 324 \\
{$[i] F_{2}$} & 2997 & 172,4 & 3016 & 2950 & 233,4 & 2933 \\
[i] $F_{3}$ & 3722 & 262,5 & 3701 & 3666 & 324,8 & 3653 \\
\hline
\end{tabular}

DP: Desvio Padrão

Tabela 4 - Teste de diferença estatística entre as médias dos grupos na vogal [i]

\begin{tabular}{c|c}
\hline Parâmetro & p valor \\
\hline$[i] f_{0}$ & $0,019^{\star}$ \\
{$[i] F_{1}$} & 0,98 \\
{$[i] F_{2}$} & 0,17 \\
{$[i] F_{3}$} & 0,27 \\
\hline
\end{tabular}

Teste de Mann-Whitney

* expressa diferença significante ao nível de 5\%

Tabela 5 - Média, mediana e desvio-padrão da frequência fundamental e das frequências dos formantes da vogal [u]

\begin{tabular}{c|ccc|ccc}
\hline \multirow{2}{*}{ Parâmetro } & \multicolumn{3}{|c|}{ Grupo Controle $(\boldsymbol{N}=\mathbf{1 0 1})$} & \multicolumn{3}{c}{ Grupo RO (N=50) } \\
\cline { 2 - 7 } & Média (Hz) & DP (Hz) & Mediana (Hz) & Média (Hz) & DP (Hz) & Mediana (Hz) \\
\hline$[\mathrm{u}] \mathrm{f}_{0}$ & 276 & 36,9 & 276 & 262 & 24,5 & 266 \\
{$[\mathrm{u}] \mathrm{F}_{1}$} & 463 & 77,1 & 464 & 491 & 95,8 & 497 \\
{$[\mathrm{u}] \mathrm{F}_{2}$} & 1838 & 1065,8 & 1298 & 2085 & 980,2 & 2564 \\
{$[\mathrm{u}] \mathrm{F}_{3}$} & 3885 & 492,1 & 3791 & 3871 & 461,8 & 3909 \\
\hline
\end{tabular}

DP: Desvio Padrão

Tabela 6 - Teste de diferença estatística entre as médias dos grupos na vogal [u]

\begin{tabular}{c|c}
\hline Parâmetro & p valor \\
\hline$[u] f_{0}$ & $0,017^{\star}$ \\
{$[u] F_{1}$} & $0,050^{\star}$ \\
{$[u] F_{2}$} & 0,30 \\
{$[u] F_{3}$} & 0,99 \\
\hline
\end{tabular}

Teste de Mann-Whitney

* expressa diferença significante ao nível de 5\%

das vias aéreas superiores, permeabilidade nasal, má-oclusão e alterações de órgãos fonoarticulatórios, resultando em diferentes procedimentos de seleção dos participantes. Tal fato é relevante uma vez que a caracterização do respirador oral a ser investigado pode apresentar reflexos nos resultados obtidos.
Em recente pesquisa1, a seleção de crianças respiradoras orais foi realizada por apenas um de três procedimentos: 1) passar menos que três minutos com um gole de água na boca; 2) apresentar vapor na face inferior isolada ou concomitante na face superior no espelho de Glatzel; 3) apresentar no mínimo três alterações orofaciais. 
Em estudos posteriores, houve um enfoque maior na caracterização da respiração oral, pois autores associaram dois procedimentos para identificação da mesma 2,16.

No presente trabalho houve uma grande preocupação com a caracterização da amostra visando minimizar os possíveis efeitos de uma seleção pouco criteriosa nos achados. Foram associados: a anamnese direcionada a queixas respiratórias, o teste de água na boca e o protocolo orofacial, sendo que para compor a amostra os participantes deveriam ser caracterizados como respiradores orais nos três procedimentos.

Em relação à análise da qualidade vocal de respiradores orais, a hipótese de que as medidas de frequências de formantes, em especial, poderiam mostrar diferenças na comparação entre vozes de respiradores orais e do grupo controle não foi confirmada. Tal hipótese foi baseada nos seguintes fatos: os formantes serem conhecidamente influenciados pela geometria do trato vocal ${ }^{25-27}$ e diversas alterações da cavidade oral terem sido apontadas como características do respirador oral ${ }^{8,10}$. Entre os testes de diferença estatística realizados, para os três primeiros formantes nas três vogais investigadas, uma única diferença estatística foi confirmada. A falta de apontamentos consistentes na literatura de alterações anatomo-fisiológicas glóticas na população de respiradores orais, conduziu à expectativa de que não seriam observadas diferenças nas medidas de frequência fundamental entre população testada e grupo controle. Entretanto, tais diferenças foram confirmadas em duas, das três vogais investigadas.

$\mathrm{Na}$ atual pesquisa as medidas de frequência fundamental apresentaram-se estatisticamente menores nas vogais $[i] \mathrm{e}[\mathrm{u}](p \leq 0,05)$ nas vozes das crianças respiradoras orais. Na vogal [a] não houve diferença estatística entre os dois grupos, apesar de ter sido observada uma tendência do grupo de respiradores orais em apresentar frequência fundamental menor, expressa pelo valor $p(p=0,057)$. Tais achados mostram que a estimação da frequência fundamental parece carregar informações a respeito do padrão respiratório inadequado de uma criança. Obviamente, a amostra do presente trabalho é insuficiente para a generalização de tais resultados.

Estudos internacionais ${ }^{20,23}$ investigaram possíveis mudanças na frequência fundamental em dois grupos de adultos em situações que corresponderiam respectivamente à respiração predominantemente oral e à nasal. Um estudo turco ${ }^{20}$ investigou um grupo de 10 adultos antes e após realização de tonsilectomia, enquanto que pesquisadores de Taiwan ${ }^{23}$ investigaram 11 adultos com obstrução nasal, antes e após o uso de descongestionante nasal (epinefrina). Os autores referiram que a frequência fundamental não foi capaz de diferenciar as vozes por eles investigadas.

Possivelmente a origem da divergência de resultados, entre os presentes achados e dos autores, esteja associada a questões metodológicas. Embora apresentem algumas semelhanças, no sentido de investigar parâmetros vocais utilizando análise acústica em situações de respiração predominantemente oral e nasal, os trabalhos guardam diferenças importantes. Um exemplo seria o fato de que, na literatura, as vozes comparadas são do mesmo participante, antes e após um determinado procedimento, enquanto que, no presente trabalho, as vozes que compõem os grupos comparados são oriundas de participantes diferentes (de faixas etárias e gênero idênticos). Por um lado os trabalhos da literatura apresentam a vantagem de excluir diferenças interpessoais, que poderiam ser apontadas como passíveis de influenciar os resultados. Por outro, a composição da amostra do presente estudo foi delineada de forma a minimizar a influência de tais diferenças nos achados, uma vez que os grupos foram caracterizados por uma sequência de procedimentos.

Outro exemplo de diferenças metodológicas importantes entre os trabalhos em questão referese ao tamanho da amostra. O presente trabalho foi desenvolvido com 50 respiradores orais e 101 participantes com respiração adequada, amostra consideravelmente mais numerosa que aquelas referidas pelos estudos realizados na Turquia ${ }^{20} \mathrm{e} \mathrm{em}$ Taiwan ${ }^{23}, 10$ e 11 participantes, respectivamente.

Apesar dos resultados deste trabalho não corroborarem os achados dos autores acima citados, os mesmos encontram suporte nos apontamentos de autores que investigaram as características anátomo-fisiológicas do respirador oral. Podem ser encontradas na literatura pesquisas que citaram que a respiração oral pode ressecar os tecidos da laringe e prejudicar a vibração das pregas vocais ${ }^{28,29}$. Outros trabalhos referiram a presença de muco excessivo e edema nas pregas vocais em quadros de alergias respiratórias ${ }^{24}$, o que poderia acarretar a diminuição da frequência fundamental ${ }^{28}$. Pesquisadores também apontaram voz mais grave como um dos sinais frequentes em pacientes alérgicos ${ }^{19}$. A associação entre obstrução nasal e o desenvolvimento de patologias laríngeas como nódulos vocais também foi referida na literatura ${ }^{29}$.

Em relação às frequências dos formantes, a investigação mostrou diferença estatisticamente significante apenas no primeiro formante $\left(F_{1}\right)$ da vogal $[u](p=0,050)$, sendo obtidos valores mais elevados no grupo de respiradores orais. Tais achados mostram que a estimação das frequências 
dos formantes não foi relevante para diferenciar o modo respiratório normal do alterado nas crianças investigadas.

A pesquisa desenvolvida na Turquia ${ }^{20}$ foi o único trabalho encontrado na literatura que investigou as frequências dos formantes em uma população com alterações que levassem a um padrão respiratório predominantemente oral.

Os presentes achados mostraram que as frequências dos formantes não foram capazes de diferenciar os grupos de respiradores orais e controle, corroborando parcialmente os achados dos autores citados. Tal grupo de pesquisa apontou discreto incremento na frequência de $F_{2}$ nas vogais [a] e [i] e decréscimo de $\mathrm{F}_{3}$ na vogal [o]. No entanto, as diferenças foram obtidas em apenas três do total de 12 medidas investigadas (associadas à frequência de formantes). As diferenças apontadas no referido trabalho parecem não ser consistentes, uma vez que não foi realizada análise estatística dos dados. Embora os achados tenham sua relevância diante da carência de pesquisas do tema na literatura, os mesmos referem-se a uma análise descritiva das observações feitas pelos autores. Pontuados pelos próprios autores, os resultados são discutíveis, sendo as palavras "incremento" e "decréscimo" referidas entre aspas. Além disso, o tamanho da amostra utilizada pelos autores (11 participantes), também representa um fator que fragiliza a consistência de seus achados.

No presente estudo, o fato das frequências de formantes não ter se mostrado relevante na diferenciação dos grupos investigados poderia ser atribuído, por um lado, a uma eventual diversidade de etiologias da respiração oral dos participantes, e por outro, a variação intersujeitos. Conforme apontado por uma autora ${ }^{21}$, diferentes etiologias da respiração oral, como hipertrofia da tonsila faríngea ou das tonsilas palatinas, podem influenciar a ressonância da voz e, portanto, possivelmente interferir na frequência dos formantes. A categorização dos respiradores orais por etiologia não foi pretendida na presente pesquisa, pois o objetivo era diferenciar respiradores orais de nasais, independente da etiologia da respiração oral. No entanto, há a possibilidade de que eventuais variações etiológicas deste modo respiratório nas crianças avaliadas possam ter influenciado a investigação dos formantes, uma vez que diferentes níveis de obstrução nasal ou faríngea podem comprometer os parâmetros acústicos pesquisados. A variação intersujeitos poderia ser apontada também como um fator que contribuiu para a ineficiência da diferenciação das frequências de formantes entre os grupos, uma vez que esse aspecto pode levar a uma variação dos formantes. Em especial, a frequência de $F_{1}$ é mais susceptível a variações intersujeitos, devido a sua relação com as diferenças anatômicas individuais na extensão do trato vocal ${ }^{30}$.

A presença de diferença estatística mostrada na frequência de $F_{1}$ da vogal [u] foi desconsiderada na conclusão dos resultados gerais, por três motivos. Primeiro, porque que se trata do único dado discrepante. Segundo, porque a significância $(p=0,050)$ se mostra em uma situação limítrofe entre a significância e não. E, finalmente, porque durante a fase de processamento, observou-se a suspeita de erro de estimação dos formantes desta vogal em particular. Outros autores também apontaram dificuldade no registro da vogal $[u]$, ao relatarem que não foi possível o registro espectrográfico da frequência do terceiro formante desta vogal em $37,7 \%$ das emissões, em uma amostra de 90 participantes ${ }^{31}$.

Uma observação complementar que pode ser feita sobre a respiração oral diz respeito ao gênero dos participantes. Estudos epidemiológicos recentes de prevalência mostram a inexistência de predominância de gênero para esse modo respiratório ${ }^{1,16}$. No entanto, apesar de cientes da falta de consistência em função do tamanho da presente amostra, considerou-se relevante comentar os achados referentes a tal questão.

Como referido na metodologia, a composição do grupo de respiradores orais, no presente estudo, foi por conveniência e, portanto não houve a preocupação em parear gêneros neste grupo. A observação de que o número de crianças respiradoras orais do gênero masculino $(76 \%)$ foi consideravelmente maior que as do feminino (24\%), corrobora os apontamentos de um grupo de pesquisadores ${ }^{3}$ sobre a tendência masculina para a respiração oral, baseados numa amostra de $64,71 \%$ de participantes do gênero masculino. Outros autores ${ }^{1}$ que concluíram a inexistência de tal prevalência destacaram que apesar de não haver encontrado diferença significante, foi observado um maior percentual de meninos respiradores orais $(53,75 \%)$ num total de 150 alunos de um projeto social. Em outro estudo 2, ao investigar 143 alunos de escolas públicas e particulares, autores apontaram mais uma vez um percentual maior no gênero masculino $(54,50 \%)$. Desta forma, observa-se que ainda não existe um consenso na literatura sobre essa questão.

\section{CONCLUSÃO}

A hipótese inicial de que as frequências de formantes seriam potencialmente capazes de diferenciar respiradores orais de nasais, em decorrência do número de alterações orofaciais descritas em respiradores orais, não foi confirmada, uma 
vez que tais medidas acústicas se mostraram ineficientes para esta tarefa.

Os achados do presente trabalho mostraram, portanto, que apenas a frequência fundamental consistiu em um parâmetro descritivo da voz que permitiu a diferenciação entre respiradores orais e crianças saudáveis. Em especial, a frequência fundamental das vogais [i] e [u] mostraram tal condição, embora a vogal [a] tenha demonstrado uma tendência em apresentar o mesmo comportamento.

Desta forma, é importante que a investigação da frequência fundamental seja um item a ser integrado à avaliação clínica da respiração oral, contribuindo na efetividade do diagnóstico. É necessário que sejam realizados novos estudos com amostras ainda mais significantes para que se possam generalizar os presentes resultados.

\section{ABSTRACT}

Purpose: to investigate spectral parameters on voices of mouth-breathing children so as to contribute to the characterization of this population, thus to an efficient diagnosis. Methods: fifty mouth-breathing children and one hundred and one nasal breathing children were selected among five and ten year old children of both genders. Recordings were obtained from carrier phrases and segments of the oral vowels [a], [i] and [u] of Brazilian Portuguese in the stressed syllable were selected to estimate the fundamental frequency $\left(f_{0}\right)$ and the frequency of the first three formants $\left(F_{1}, F_{2}\right.$ e $\left.F_{3}\right)$. The signals were recorded, cut out and processed using Praat software. Results: the fundamental frequency for the vowels $[i]$ and $[u]$ showed lower frequency in the mouth-breathing group, while the frequency $F_{1}$ of the vowel $[\mathrm{u}]$ was higher in the same group. Conclusion: our findings pointed out that the fundamental frequency is a vocal parameter that helps us to differentiate mouth-breathing children from nasal breathers, therefore contributing for a mouth breathing classification system.

KEYWORDS: Mouth Breathing; Voice; Signal Processing, Computer-Assisted; Child; Speech Acoustics

\section{REFERÊNCIAS}

1. Menezes VA, Leal RB, Pessoa RS, Pontes RMES. Prevalência e fatores associados à respiração oral em escolares participantes do projeto Santo AmaroRecife, 2005. Rev Bras Otorrinolaringol. 2006; 72(3):394-9.

2. Menezes VA, Leal RB, Moura MM, GranvilleGarcia AF. Influência de fatores socioeconômicos e demográficos no padrão de respiração: um estudo piloto. Rev Bras Otorrinolaringol. 2007; 73(6):826-34.

3. Di Francesco RC, Passerotti G, Paullucci B, Miniti A. Respiração oral na criança: repercussões diferentes de acordo com o diagnóstico. Rev Bras Otorrinolaringol. 2004; 70(5):665-70.

4. Mahony D, Karsten A, Linder-Aronson S. Effects of adenoidectomy and changed mode of breathing on incisor and molar dentoalveolar heights and anterior face heights. Aust Ortod J. 2004; 20(2):93-8.

5. Mattar, SEM, Anselmo-Lima WT, Valera FCP, Matsumoto MAN. Skeletal and occlusal characteristicsinmouthbreathingpre-schoolchildren. J Clin Pediatr Dent. 2004; 28(4):315-318.

6. Côrrea ECR, Bérzin F. Mouth breathing syndrome: cervical muscles recruitment during nasal inspiration before and after respiratory and postural exercises on Swiss Ball. Inter J Pediatr Otorhinolaryngol. 2008; 72(9):1335-43.

7. Lemos CM, Wilhelmsen NSW, Mion OG, Júnior JFM. Alterações funcionais do sistema estomatognático em pacientes com rinite alérgica: estudo caso-controle. Rev Bras Otorrinolaringol. 2009; 75(2):268-74.

8. Motonaga SM, Berte LC, Anselmo-Lima WT. Respiração bucal: causas e alterações no sistema estomatognático. Rev Bras Otorrinolaringol. 2000; 66(4):373-9.

9. Di Francesco RC. Crescimento craniofacial e distúrbios da respiração oral do ponto de vista otorrinolaringológico. In: Krakauer, LH, Di Francesco RC, Marchesan IQ. Conhecimentos essenciais para atender bem a respiração oral. São José dos Campos: Pulso Editorial; 2003.p. 27-35. 
10. Andrade FV, Andrade DV, Araújo AS, Ribeiro ACC, Deccax LDG, Nemr K. Alterações estruturais de órgãos fonoarticulatórios e más oclusões dentárias em respiradores orais de 6 a 10 anos. Rev. CEFAC. 2005; 7(3):318-25.

11. Bianchini AP, Guedes ZCF, Vieira MM. Estudo da relação entre a respiração oral e o tipo facial. Rev Bras Otorrinolaringol. 2007; 73(4):500-5.

12. Lessa FCR, Enoki C, Feres MFN, Valera FCP, Lima WTA, Matsumoto MAN. Influência do padrão respiratório na morfologia craniofacial. Rev Bras Otorrinolaringol. 2005; 71(2):156-60.

13. Cattoni DM, Fernandes FDM, Di Francesco RC, Latorre MRDO. Características do sistema estomatognático de crianças respiradoras orais: enfoque antroposcópico. Pró-Fono. 2007; 19(4):347-51.

14. Sígolo C, Silveira M, Quintal M, Sakano E, Tessitore A. Ocorrência de movimentos primários de língua em crianças respiradoras oronasais. Rev. CEFAC. 2008; 10(1):51-7.

15. Marchesan IQ. Protocolo de avaliação miofuncional orofacial. In: Krakauer LH, Di Francesco RC, Marchesan IQ. Conhecimentos essenciais para atender bem a respiração oral. São José dos Campos: Pulso Editorial; 2003. p. 55-79.

16. Abreu RR, Rocha RL, Lamounier JA, Guerra AFM. Prevalência de crianças respiradoras orais. J Pediatr. 2008; 84(5):467-70.

17. Almeida FL, Silva AMT, Serpra EO. Relação entre má oclusão e hábitos orais em respiradores orais. Rev. CEFAC. 2009; 11(1):86-93.

18. Tavares JG, Silva EHAA. Considerações teóricas sobre a relação entre respiração oral e disfonia. Rev Soc Bras Fonoaudiol. 2008; 13(4):405-10.

19. Corsi SL, Castro FFM, Antila MA, Behlau $M$. Incidência de sinais e sintomas vocais em pacientes com rinite alérgica. In: Behlau M. A voz do especialista. vol 2. Rio de Janeiro: Revinter; 2001.p. 89-100.

20. Ilk HG, Erogul O, Satar B, Özkaptan Y. Effects of tonsillectomy on speech spectrum. J Voice. 2002; 16(4):580-6.
21. Denunci, FV. Respiração Oral e Qualidade Vocal na Infância: um estudo Comparativo. [dissertação] São Paulo (SP): Pontifícia Universidade Católica de São Paulo; 2003.

22. Beneton G, Botelho SW, Silva L, Castro, PC. Comparação dos parâmetros vocais em crianças com e sem rinite alérgica. Rev Fono Atual. 2004; 7(29):30-5.

23. Lee GS, Yang CCH, Wang CP, Kuo TBJ. Effect of nasal decongestion on voice spectrum of a nasal consonant-vowel. J Voice. 2005; 19(1):71-7.

24. Simberg S, Sala E, Tuomainen J, Rönnemaa AM. Vocal symptoms and allergy: a pilot study. J Voice. 2009; 23(1):136-9.

25. Behlau M, Madazio G, Feijó D, Pontes P. Avaliação de voz. In: Behlau M. Voz: o livro do especialista. vol.1. Rio de Janeiro: Revinter; 2001. p. $85-176$.

26. Magri A, Cukier-Blaj S, Karman DF, Camargo ZA. Correlatos perceptivos e acústicos dos ajustes supraglóticos na disfonia. Rev. CEFAC. 2007; 9(4):512-8.

27. Lima MFB, Camargo ZA, Ferreira LP, Madureira, S. Qualidade vocal e formantes das vogais de falantes adultos da cidade de João Pessoa. Rev. CEFAC. 2007; 9(1):99-109.

28. Pinho SMR. Avaliação e tratamento da voz. In: Pinho SMR. Fundamentos em fonoaudiologia. Tratando os distúrbios da voz. 2. ed. Rio de Janeiro: Guanabara Koogan; 2003. p. 3-40.

29. Martins RHG, Trindade SHK. A criança disfônica: diagnóstico, tratamento e evolução clínica. Rev Bras Otorrinolaringol. 2003; 69(6):801-6.

30. Figueiredo RM. Identificação de falantes: aspectos teóricos e metodológicos. [tese]. Campinas (SP): Universidade Estadual de Campinas; 1994.

31. Behlau MS, Pontes PAL, Ganança MM, Tosi O. Análise espectrográfica de formantes das vogais do português brasileiro. ACTA AWHO. 1988; 7(2):74-85.

DOI: 10.1590/S1516-18462010005000107

RECEBIDO EM: 02/10/2009

ACEITO EM: 27/05/2010

Endereço para correspondência:

Danieli Viegas

Rua Chaves Pinheiro, 58 sl. 101

Rio de Janeiro - RJ

CEP: 20771-470

E-mail: fdviegas@gmail.com 